française

\section{La Révolution française}

Cahiers de l'Institut d'histoire de la Révolution française

$20 \mid 2021$

La Révolution en 3D - Textes, images, sons

(1787-2440)

\title{
Narrativité, rupture et révolution
}

Jean-Claude Milner

\section{OpenEdition}

Journals

Édition électronique

URL : https://journals.openedition.org//rf/4863

DOI : $10.4000 / / r f .4863$

ISSN : 2105-2557

Éditeur

IHMC - Institut d'histoire moderne et contemporaine (UMR 8066)

Référence électronique

Jean-Claude Milner, « Narrativité, rupture et révolution », La Révolution française [En ligne], 20 | 2021, mis en ligne le 25 juin 2021, consulté le 28 juin 2021. URL : http://journals.openedition.org/lrf/4863 ; DOI : https://doi.org/10.4000/Irf.4863

Ce document a été généré automatiquement le 28 juin 2021.

(c) La Révolution française 


\title{
Narrativité, rupture et révolution
}

\author{
Jean-Claude Milner
}

\section{NOTE DE L'ÉDITEUR}

Cet article est basé sur une communication présentée lors du colloque « La Révolution en 3D - Textes, images, sons (1787-2440) » qui s'est tenu à l'université Paris 1 PanthéonSorbonne du 14 au 16 mars 2019, organisé par le Cespra et l'IHMC-IHRF. Vous pouvez retrouver cette communication sur la chaîne YouTube de l'IHMC à l'adresse : https:// youtu.be/90DSe_oNXrM

1 Je dois avouer que j'ai peu pratiqué les travaux d'Alphonse Aulard. Néanmoins le hasard a voulu que j'aie lu assez tôt sa critique de Taine ${ }^{1}$. Moi-même lecteur et admirateur des Origines de la France contemporaine, je n'en avais pas moins été enthousiasmé par le sérieux de l'examen ; j'y avais en particulier noté un attachement au premier devoir du chercheur: ne pas faire obstacle aux chercheurs suivants. Devoir que, selon Aulard, Taine n'avait pas respecté, tant par le désordre inextricable qu'il laissait dans les archives après les avoir consultées, que par la désinvolture dans le choix de ses références. La sévérité n'excluait pas le sourire; du réquisitoire naissait un chef d'œuvre de cette forme rare d'humour que j'appellerai l'humour académique. Mais l'humour ne doit pas dissimuler la gravité; quand les apparences de la précision servent à installer l'invérifiable, une faute irréparable est commise. Cet avertissement d'Alphonse Aulard, je l'ai pris au premier chef pour moi ; en ai-je toujours suffisamment tenu compte, je l'ignore, mais je l'espère.

\section{1 - La Révolution française et le récit}

La Révolution française et le récit, la question se pose pour deux raisons au moins.

3 L'événement a transformé le récit de prose dans la langue française ${ }^{2}$. Après la Révolution et l'Empire, commence, dans la littérature, le règne du roman-roi; simultanément, la transformation sociale passe désormais pour l'affaire majeure de 
l'humanité. Au confluent de ces deux mutations, la transformation sociale devient justement l'objet privilégié du roman français. Le présent y est saisi entre un passé, dont parfois on ne sait rien sauf qu'il n'est plus, et un avenir, tantôt déjà là (et pour le pire), tantôt indéterminable et ouvrant une temporalité indéfinie. Nostalgie ou inquiétude, ce que la critique a baptisé « réalisme» repose sur ce doublet. Il s'agit moins de la réalité sociale en tant que telle que de son perpétuel déséquilibre, conséquences ou prémices du changement. Il arrive que le romancier préfère projeter ce déséquilibre dans l'intériorité des psychologies; la loi du réalisme n'en est pas affectée. Le roman réaliste se veut grand récit de la transformation sociale.

Dans Le Temps retrouvé, Proust résume la donnée matérielle qui depuis Balzac déterminait le roman : "Ce qui caractérisait le plus cette société, c'était sa prodigieuse aptitude au déclassement. » Déclassement, le lecteur moderne peut s'y tromper; il n'est pas question seulement de l'abaissement, mais de toute forme de mobilité. Quand Bloch connaît des succès mondains sous le nom de Du Rozier, il s'élève, mais il ne se déclasse pas moins que Charlus, abaissé par les Verdurin. Aptitude au déclassement, on ne saurait mieux paraphraser, en termes neutres, la lutte des classes. Proust n'en fera pas son unique objet, rompant ainsi, en pleine conscience, avec Balzac, mais il sait qu'elle continue de former le fond de son entreprise. Je propose l'hypothèse suivante: reconnaître ici les traces de l'expérience qui a traversé la société française, à partir de 1789, et dont l'Europe entière a été marquée.

Que le récit soit déterminé par la transformation sociale, cela va si peu de soi que l'on a pu refuser cette possibilité et faire du roman la narration du fixisme social. La question s'est posée quand le récit romanesque commença de rejeter ouvertement le roman réaliste au profit des intermittences du cœur. La psychologie, devenue seule mobile, se disjoint de la société, dont la description évanouissante privilégie les immobilités. De ce point de vue, il n'est pas surprenant que la NRF, se détournant du « roman réaliste » ait porté aux nues le grand narrateur du fixisme social que fut Simenon. On comprend aussi qu'en un premier mouvement, elle ait mis Proust à l'écart. Sans doute faudrait-il ici rappeler la doctrine des pères fondateurs de la IIIe République; quoiqu'ils l'aient exprimée au sortir de la Première guerre mondiale, elle les habitait depuis la victoire des dreyfusards; elle est rapportée par Brice Parain : «En France, rien ne changera. D'ailleurs il n'y a rien à changer ${ }^{3}$. " Considérer à la lumière de ce renoncement la configuration présente de l'opinion publique et l'état de la littérature hexagonale, cela aurait son prix, mais nous éloignerait trop de notre propos.

6 Car une autre donnée doit être mise en lumière. C'est que la Révolution et l'Empire ont d'emblée demandé à être racontés. Ils demandent le récit et même le grand récit. J'entends par là un récit qui ne se contente pas de narrer la séquence événementielle d'une période, mais se fixe au moins quatre tâches supplémentaires : a) mettre au jour à la fois l'unité et l'unicité de la séquence choisie ; b) déterminer les causes initiales de la séquence, en distinguant, à l'exemple de Thucydide, causes apparentes et causes effectives ; c) établir que la séquence est à l'origine de nouveautés radicales dans les actions et les discours; d) identifier ces nouveautés. Rien ici ne va de soi et surtout pas le fait que la Révolution et l'Empire se soient prêtés si vite à des récits qui entendent répondre à de telles exigences, alors que d'autres moments de l'histoire de France ne s'y prêtent pas. Un détour par Claude Lévi-Strauss permet d'illustrer le contraste. On se souvient que celui-ci, à la fin de La Pensée sauvage, commente le récent livre de Sartre Critique de la Raison dialectique. Il le ramène à la question: "À quelles conditions le 
mythe de la Révolution française est-il possible ${ }^{4}$ ?» Pour argumenter sa position, il prend l'exemple de la Fronde. Je ne voudrais pas entrer dans le détail de la doctrine de Lévi-Strauss, bien qu'il mérite d'être repris plus qu'attentivement. Je m'en tiendrai au minimal : par mythe, selon moi, est ici désigné un récit qui fonctionne comme schème d'interprétation de tout événement possible. Pour peu que l'événement soit tenu pour grand, le récit interprétatif sera grand, lui aussi ; la plupart des mythes amérindiens analysés par Lévi-Strauss forment de grands récits ou des fragments de grands récits. Le dispositif a sa réciproque : il y a mythe, selon Lévi-Strauss, dès que les schèmes d'interprétation ultimes d'un événement prennent la forme d'un grand récit. Et de fait on peut soutenir qu'encore récemment, sous nos yeux, un événement comme les gilets jaunes fut, par certains, passé au filtre de la prise de la Bastille, de Marie-Antoinette, de l'affaire du collier. Tant le mythe de la Révolution française a conservé de force. Au fond, le geste lévi-straussien consiste à formuler une réversibilité systématique entre mythe et récit. Tant comme Historie que comme Geschichte, l'histoire devient la matrice de production des mythes dans les sociétés dites chaudes. Ainsi devient-il possible à l'anthropologie de mettre à la question toute historisation d'un événement et tout événement historisable.

7 Je répète que je ne discuterai pas cette approche, même si elle a pu autoriser d'importants développements ultérieurs. Je m'en tiens à une seule question : pourquoi la Fronde surgit-elle dans l'argumentation?

8 La raison doit sauter aux yeux : il s'agit d'un événement historique qui justement ne fait pas mythe. Je traduis: un grand événement sans grand récit ${ }^{5}$. Car une remarque s'impose: la Fronde a, selon certains historiens modernes, je pense à Jean-Marie Goulemot, entraîné un traumatisme profond, au point que l'absolutisme est alors apparu, pour plus d'un siècle encore, le seul rempart efficace contre le retour d'événements semblables ${ }^{6}$. L'émergence d'un grand récit n'aurait rien eu de surprenant. Elle ne se produisit pas; en son lieu et place, se succédèrent les éloges du Roi, aussi dispersés et brillants qu'une pluie d'or. La Fronde ne sera pas racontée. Les mémoires de Retz seront publiés après la mort de Louis XIV, à un moment où le récit ne prête plus à conséquence. Il en va de même des mémoires de Madame de Motteville. Quant aux années qui ont suivi la Fronde, les fameuses années 1660 qui ouvrent le classicisme français, elles se vouent au silence sur le sujet. Louis XIV fait brûler, dans les archives du Parlement de Paris, les documents de la période et il apparaît dans Tartuffe que le seul fait, pour un particulier, de conserver des papiers concernant ce que Molière appelle "nos troubles ", peut entraîner une dénonciation, dont les effets, sans l'intervention quasi-magique du Roi, seraient destructeurs, non seulement pour le coupable, mais pour toute sa famille ${ }^{7}$. Précisément à cause de son importance, la Fronde ne fera pas récit, tel est le mot d'ordre secret de notre littérature classique; on ne la trouvera qu'au détour des Fables de La Fontaine ou en arrière-fond des Maximes de La Rochefoucauld ou dans quelques détails dissimulés des comédies de Molière. On peut parler de refoulement ; l'initiateur en fut Louis XIV, talentueux concepteur de pompes refoulantes. En 1734, Voltaire décrit les frondeurs, dans la huitième de ses Lettres philosophiques: « des écoliers qui se mutinent contre le préfet du collège et qui finissent par être fouettés »; quand il publie Le Siècle de Louis XIV en 1751, on peut conclure que le mythe du grand règne l'a définitivement emporté sur tout autre récit possible.

9 Pour la France contemporaine, des exemples analogues se proposent. Nous pouvons dire qu'il existe un grand récit de la Résistance; certes il n'est plus accepté sans 
réserves, mais il fonctionne encore comme point de départ, y compris et peut-être surtout pour ceux qui souhaitent le corriger. Mais la fin de la IIIe République en 1940 ? Mais la collaboration? Je maintiens que nous n'en avons pas de récit qui soit à la mesure des conséquences ; celles-ci pèsent encore aujourd'hui sur le référent national français. Et qu'en est-il de la guerre d'Algérie ? Ou de Mai 68 ? Là encore le refoulement est à l'œuvre. Paradoxalement, un grand récit de De Gaulle commence à se construire. Il inclut la Ve République; pourtant le coup d'état de 1958 y demeure innomé et le pamphlet de François Mitterrand, en adjoignant le qualificatif permanent au substantif coup d'état, fait très précisément et très volontairement obstacle à tout récit. On peut donc soutenir que la Fronde, la fin de la IIIe République, la prise du pouvoir par Pétain, la collaboration, la guerre d'Algérie, 1958, Mai 68 refusent le récit. Même dans le cas de la Fronde que l'on pourrait croire bien lointaine, ces refus valent refoulement et déterminent notre présent par leur absentement.

Par contraste, on mesure mieux l'étonnante singularité de la Révolution et de l'Empire. Ils ont demandé très tôt le récit. Tant du côté de leurs partisans que du côté de leurs adversaires. Tant du côté des acteurs que des historiens ultérieurs. Les récits et contrerécits ont été multiples parce que, des années 1789-1815, aucun groupe de vainqueurs ne sortit, comparable au Parti communiste bolchevique ou au Parti communiste maoïste. Or, quand on dit que l'histoire est faite par les vainqueurs, on sous-entend que ces derniers s'approprient le monopole du récit. Pour peu cependant qu'il n'y ait pas de vainqueurs constitués en un seul groupe, la pluralité des récits règne.

11 Cette pluralité n'a pourtant pas empêché la montée en puissance des grands récits. Autre singularité donc : autour de la Révolution française s'est formé non seulement un essaim de récits, mais bien un essaim de grands récits, rivaux les uns des autres. Pour ne prendre qu'un seul exemple, Aulard tenait à disqualifier le récit de Taine qui, luimême, avait entrepris de réfuter les récits antérieurs; on sait que le récit d'Aulard n'a pas tardé à être rejeté. Or, une telle rivalité ne va pas de soi, surtout quand il s'agit des révolutions.

\section{2 - Les révolutions, du récit unique à l'annulation du récit}

$12 \mathrm{Au} \mathrm{xx}^{\mathrm{e}}$ siècle, la révolution soviétique a longtemps dépendu d'un seul grand récit, par rapport auquel tous les autres ont paru fragiles, circonstanciels, non-concluants. La révolution cubaine a suivi la même voie. En sorte qu'aujourd'hui, alors que l'URSS a disparu et que Fidel Castro s'est retiré, on peut se demander s'il subsiste quelque récit que ce soit ou seulement des fragments précaires. La Chine a connu des processus tout autres ; après avoir connu le grand récit unique, rédigé par le parti unique, elle n'a pas cessé de le contester toujours davantage. Mao Zedong lança l'offensive par sa circulaire du 16 Mai 1966, confirmée et renforcée par son fameux dazibao du 5 Août, intitulé « Feu sur le quartier général». Ainsi commença la Grande révolution culturelle prolétarienne. Un de ses premiers objectifs fut d'abattre les héros du grand récit; au premier rang d'entre eux, on trouvait Liu Chao-Chi (Liu Shaoqi). Il ne survécut pas.

13 Mao prit l'initiative, mais Lin Piao, proclamé second du Grand Timonier, infléchit le parcours, en s'appuyant sur ceux qu'on appellera plus tard la bande des Quatre : Jiang Qing, très influente épouse de Mao; Zhang Chunqiao; Yao Wenyuan, brillant 
intellectuel à la plume acérée ; Wang Hongwen. Mao, en effet, s'en prenait aux héros du grand récit, mais le grand récit lui-même ne le gênait pas, tant qu'il en était l'ordonnateur. Or, la révolution culturelle alla plus loin; l'intégralité du geste révolutionnaire étant ramenée à la seule lutte entre l'ancien et le nouveau, tout ce qui se stabilise s'inscrit, de ce fait même et dans l'instant, comme ancien; pour ce motif, il doit disparaître sans que lui soit laissée la moindre possibilité de retour. Cela vaut pour cette forme de permanence qu'est la survie d'un être humain : l'idéologie de la survie est dénoncée comme source de toutes les dérives contre-révolutionnaires. La même condamnation pèse sur la permanence qu'assure une transmission. Naguère la tradition révolutionnaire requérait un devoir de transmettre ; très logiquement, elle privilégiait la fixation d'un grand récit ; tout ce dispositif hérité est désormais balayé.

On peut admettre que l'ensemble intégral des stabilisations discursives se définisse comme culture; dès lors, on mesure la portée du prédicat culturel, attribué à la révolution. La révolution culturelle vise la forme culture comme telle; pour ce faire, elle doit commencer par abolir la forme récit. Seuls seront admis les souvenirs des malheurs passés, tels que les victimes directes les racontent, sous forme d'anecdotes dispersées. Ce qui est vrai de tout récit, l'est a fortiori du grand récit de la grande révolution prolétarienne; justement parce qu'il est grand, celui-ci porte sa fixité au degré maximal. Le respect même qu'il inspirait naguère témoigne du danger qu'il distille à jamais. Après tout, le titre et la disposition interne du Petit livre rouge révèlent le secret: au grand se substitue le petit, le continu des discours et articles est remplacé par le discontinu des extraits, le nom propre de Mao Zedong absorbe toute narrativité autre qu'anecdotique, la possibilité de quelque grand récit que ce soit est abolie à jamais, comme est du reste abolie la consistance doctrinale. La toute-puissance de la doctrine de Marx est effacée par la pensée-Mao Zedong ; cette dernière se réduit au prononcé du nom propre Mao Zedong et au pur geste de brandir le Petit livre rouge.

Par sa nature même, la Révolution culturelle ne pouvait laisser que des traces documentaires dispersées: brochures, articles du Quotidien du Peuple et de Pékin Informations. Qui plus est, le non-sinologue travaille sur les traductions autorisées par le pouvoir politique du moment. Selon moi, l'obstacle n'est pas insurmontable, loin de là. Paradoxalement, la propagande propose ici une source digne de foi. En tant qu'officielles, les traductions reflétaient l'interprétation que les responsables de la Révolution culturelle se formaient de leur propre action. Je n'ai fait qu'en proposer une synthèse. Quant aux actes commis au cours de la Révolution culturelle, on comprend aisément qu'étant admis l'extension proprement illimitée du «culturel », aucun détail, si menu ou si global soit-il, de la vie quotidienne ou de l'organisation sociale d'ensemble ait pu échapper à la vigilance révolutionnaire.

16 Lin Piao fut éliminé en 1971. La Bande des Quatre demeura en place, mais son influence diminua, à mesure que Zhou Enlai consolidait la sienne, en luttant de plus en plus ouvertement contre les conséquences matérielles de la Révolution culturelle. Il apparut ainsi comme un recours et sa mort, en janvier 1976, fut marquée par des manifestations de colère contre la Bande des Quatre. Un véritable mouvement d'opposition se déclare en avril. La lutte entre les factions éclate au grand jour. Mao meurt en octobre 1976 ; un mois après, les Quatre sont arrêtés. Le Parti ne dissimula plus alors que la révolution culturelle se concluait sur des ruines et des cadavres. Néanmoins, la forme récit ne revint pas. Ni en Chine, ni ailleurs, on ne sait narrer la Grande révolution culturelle prolétarienne; que Mao ait pu, grâce à elle, triompher de ses adversaires, on le 
constate, mais le détail de sa tactique et de sa stratégie demeure obscur. Manifestement, la reconstruction de la Chine s'est vouée de plus en plus ouvertement à la forme marchandise, or cette forme n'a rien à raconter. Bien au contraire, elle s'étend sans rupture comme une eau qui noie toutes les ruptures. A la mort de Mao, Teng Siao Ping prit acte du tournant à accomplir, mais en prenant bien soin de se présenter en continuateur de Mao. Ainsi vouait-il au secret la rupture qu'il incarnait. La dénonciation de la Bande des Quatre se substituait à la narration; seul demeurait, encore une fois, le rappel des malheurs individuels. Il revint aux romans et aux films de montrer des fragments de ce qui avait eu lieu. Confrontée aux lacunes de ce qui lui reste du grand récit révolutionnaire, la Chine des marchandises et des techniques semble ne pouvoir retrouver la forme récit qu'en recourant aux grands récits et légendes de l'Empire du Milieu.

Au risque de me répéter, j'insiste. La Révolution française a ceci de singulier : non seulement elle demande le récit, mais très vite, les récits multiples se projettent dans la forme du grand récit, sinon qu'une multiplicité de grands récits se combattent. Par cette multiplicité, elle s'oppose à la Révolution soviétique ; en maintenant, très avant dans le $\mathrm{xx}^{\mathrm{e}}$ siècle, la forme du grand récit, elle s'oppose à la Révolution chinoise.

\section{3 - Splendeurs et misères du grand récit}

Tout en n'adhérant ni aux prémisses ni aux conclusions de François Furet, je comprends le mouvement qui l'anima et dans une certaine mesure, je le partage : face à la rivalité indéfinie des grands récits, il entreprend d'y échapper en refusant le grand récit. Ce refus conditionne, selon lui, la possibilité de la pensée. C'est pourquoi Penser la Révolution française (1978) propose un recueil de micro-récits, dont chacun est étroitement circonscrit dans le temps et contient, il faut bien l'avouer, peu de pensée ; seule la préface unifie l'ensemble de manière à répondre au titre ${ }^{9}$. C'est pourquoi le véritable complément de ce livre porte sur le fondement ultime du grand récit des révolutions. Le Passé d'une illusion (1995) dénonce des contre-vérités, des dissimulations concernant les faits du passé soviétique ${ }^{10}$; ce faisant, l'auteur semble substituer un nouveau grand récit à l'ancien, mais en vérité, il construit une machine de guerre contre la forme du grand récit comme telle. À l'image d'un infiltré qui entre au sein d'un réseau afin de le démanteler, Furet adopte la forme du grand récit pour détruire la légitimité de tout grand récit possible. L'illusion dénoncée comme passée dans le titre, c'est le grand récit lui-même.

On ne s'étonnera donc pas que le monument de l'école Furet se matérialise en un Dictionnaire critique de la Révolution française, bien fait pour annoncer au monde qu'il n'y aura plus de place désormais, sous le soleil de la raison, pour un grand récit de la Révolution française ${ }^{11}$. L'abolition commence avec la forme du dictionnaire; elle se poursuit avec la pluralité des auteurs : deux maîtres d'œuvre, François Furet et Mona Ozouf, et un grand nombre de collaborateurs. Le titre de l'ouvrage se dévore lui-même : si l'on peut écrire un dictionnaire de la Révolution française, comme on a écrit un dictionnaire de La Comédie humaine, cela implique que la première a le même mode d'existence que la seconde. Nul n'ignore que celle-ci n'a d'autre réalité que les romans ou nouvelles qui la composent. Bien rares ceux qui en ont lu l'intégralité, mais euxmêmes n'ont eu accès qu'aux parties constitutives et non au tout, qui recule indéfiniment à l'horizon. Tout comme La Comédie humaine, la Révolution française, 
selon cette approche, n'est qu'un nom donné à un amoncellement de parcelles. $\mathrm{Si}$, de plus, le dictionnaire est critique, on conclura que la Révolution française au singulier défini n'a pas d'autre mode d'existence que le grand récit biblique, après le travail de la Hochkritik: une mosaïque de fragments, de nature diverse, issus de sources contradictoires et inconciliables entre eux.

À vrai dire, les trois lexèmes la, Révolution, française ne forment pas même un nom aux yeux d'un disciple strict de l'école Furet. Ils recomposent plutôt un aide-mémoire, comparable au signifiant Ornicar dans la liste des conjonctions " mais où est donc Ornicar? ». Ce trisyllabe, en droit, ne dénomme rien ni personne ; il fonctionne certes comme un groupe nominal, sujet du verbe être, mais à des fins de pure commodité. De la même manière, François Furet consent, par compassion pour les esprits faibles, à feindre d'accepter le nom Révolution française.

21 Je ne garantirai pas que cette position ait été partagée par tous ses collaborateurs et singulièrement par Mona Ozouf. Il n'est pas impossible que celle-ci ait toujours cru au réel unitaire de la Révolution française, sinon que ce réel ne serait pas saisissable dans la forme du grand récit ${ }^{12}$.

22 La Révolution française serait alors une hypothèse, donnant sens et orientation à des travaux, dont certains pourraient prendre la forme du récit, mais de récits partiels. La narrativité totalisante n'existerait que pour faciliter la transmission du savoir historique auprès des apprenants. Le grand récit ne serait au finale, pour reprendre une expression de Michel Foucault, rien de plus qu'une " petite pratique pédagogique ». Certains ultra-pédagogues semblent s'en être convaincus; soucieux de préserver leur expertise pédagogique contre tout soupçon d'attachement aux petites pratiques, ils souhaitent bannir le syntagme « Révolution française » loin des programmes scolaires.

23 En usant de l'expression grand récit, je me suis borné jusqu'à présent à lui accorder une portée descriptive. En cela, j'ai suivi l'exemple de la recherche de langue française, qui a fait du grand récit une donnée d'évidence ${ }^{13}$. On commence à entrevoir que cette évidence peut être rejetée ; il ne s'agit plus seulement de décomposer le grand récit de la Révolution française, mais de décomposer tout grand récit comme tel. Le moment est donc venu d'apporter quelques précisions critiques à un débat encore naissant. L'expression grand récit elle-même a été thématisée d'abord par Jean-François Lyotard ${ }^{14}$ et a donné naissance à un véritable champ de recherches, notamment aux États-Unis. Il convient d'en séparer deux acceptions; l'une insiste sur la fonction de légitimation, dont la narrativité est un moyen parmi d'autres; l'autre met en avant la narrativité, dont la légitimation est une conséquence parmi d'autres. Lyotard illustre la première voie; chez lui, le grand récit prend rang parmi les discours de légitimation des pratiques, des savoirs, des institutions. De tels «métadiscours » ne se constituent pas nécessairement en récits ; quand ils le font, on parle de "métarécits » : de ce fait, les expressions grand récit et métarécit sont strictement synonymes. Lyotard donne en exemple le récit des Lumières, le récit de l'émancipation du travailleur, le récit du progrès. Même si le post-moderne se définit par sa méfiance à l'égard des métarécits, ceux-ci ne sont pas traités en adversaires à combattre.

Chez Michel Foucault et ceux qui le suivent, la seconde acception l'emporte ${ }^{15}$. Loin de se situer dans l'espace surplombant qu'ouvre le préfixe méta-, le grand récit se dispose dans une topologie sans haut, ni bas; il est toujours-déjà donné et, réciproquement, le déjà donné tend toujours à se conformer en récit. Plutôt qu'une légitimation de droit, il faut le tenir pour une matrice quasi-matérielle, permettant la formation de récits 
dérivés. On peut relire à cette lumière la citation de René Char qui clôt chacun des tomes de L'Histoire de la sexualité: «L'histoire des hommes est la longue succession des synonymes d'un même vocable. Y contredire est un devoir ». La longue succession synonymique, ici dénoncée, qu'est-ce donc sinon la forme la plus générale du grand récit? "Y contredire", un tel principe éthique va bien au-delà de la méfiance lyotardienne.

Dans ces conditions, on comprend que la Révolution française n'apparaisse affirmativement ni dans l'Histoire de la folie, ni dans Les Mots et les choses, ni dans Surveiller et punir, ni dans l'Histoire de la sexualité. Cette absence ne doit rien au travail de Furet quoiqu'elle s'y accorde; elle appartient intégralement au programme de Foucault : la Révolution française vaut immédiatement grand récit; tout grand récit vaut réitération d'un même synonyme; pour en contrer le dire, l'action la plus efficace consiste dans un silence. Le même effacement se constate pour la révolution galiléenne, proprement démantelée dans Les Mots et les choses ${ }^{16}$, ou pour le Capital de Marx, ramené à une variante combinatoire de Ricardo : la critique de l'économie politique tombe aux oubliettes, réduite à une variation interne à l'économie politique elle-même ${ }^{17}$. De fait, révolution, Galilée, Marx et bien d'autres noms de l'histoire encapsulent, comme ces bois japonais qui se déploient en fleurs, un ou plusieurs grands récits. Que ceux-ci légitiment des pouvoirs-savoirs, sans doute, mais en tant que récits, ils importent davantage par leur volonté d'inscrire tout geste possible dans la forme de la geste. Pour cette raison et nulle autre, ils ne sauraient échapper à leur mise en examen. Selon Foucault, tout grand récit doit être dénoncé ; doit être parallèlement décomposé tout nom qui porte en soi, comme un vice caché, la possibilité d'un grand récit ${ }^{18}$.

On mesure à cette occasion que la fugitive sympathie de Foucault pour les maoïstes français dépendait de l'attachement de ces derniers pour la révolution culturelle; elle ne se prolongea d'ailleurs pas après la chute de Lin Piao, justement parce que la mise en écho dépendait du rejet, par Lin Piao et son groupe, de la culture comme espace de synonymie et du grand récit comme ressassement d'un même synonyme. De même, Foucault crut percevoir dans la révolution des mollahs iraniens une main parricide levée contre les deux grands récits européens des révolutions et des Lumières. Il fut moins séduit par les affirmations des mollahs qu'il ne se sentit conforté par leurs refus. Les imaginant consonants au sien, il eut la faiblesse de prendre cette homonymie de circonstance pour une coappartenance structurale.

\section{4 - Le grand récit, inévitable et impossible}

Alexandre Koyré avait isolé certains termes qu'on n'applique jamais qu'aux autres et jamais à soi-même ; ainsi en allait-il, selon lui, du terme " hérétique » : ce sont eux qui sont hérétiques, nous sommes orthodoxes ${ }^{19}$. De même, le grand récit est toujours censé venir des autres, que ce soit chez Lyotard ou chez Foucault. Mais un moment arrive où l'on entend, prononcé contre soi-même, le fatidique de te fabula narratur. On examinera quelque jour si Foucault n'a pas lui-même écrit, dans l'Histoire de la sexualité, le grand récit de l'intériorisation, par le christianisme augustinien, de l'acte sexuel en désir (ou libido, terme d'Augustin) ${ }^{20}$. De même, François Furet a-t-il peut-être contribué au grand récit de la collapsologie politique, né de la surprise que provoquèrent, auprès des sexagénaires de la dernière décennie $d u x^{e}$ siècle, les échecs des révolutions exemplaires et la catastrophe finale de l'URSS. Quant à la méfiance que 
Lyotard opposait aux grands récits, d'aucuns y discernent une posture occidentale, où ne se symptomatiserait rien d'autre que le déclin de l'hégémonie matérielle et discursive de l'Occident ${ }^{21}$. Tout bien considéré, la Révolution culturelle chinoise avait raison sur un point : toutes les sociétés tendent à perpétuer indéfiniment les discours qu'elles produisent; cette forme d'immobilité s'appelle une culture. À moins de détruire sans reste monuments et archives, à moins de mettre à mort les porteurs de la plus petite parcelle de passé, une culture toujours s'installe, tantôt par volonté tantôt par inertie. Il suffit que quelques-uns, si peu nombreux qu'ils soient, se souviennent, si peu que ce soit, d'une combinaison de vocables, si perturbatrice qu'elle se soit voulue, pour qu'un récit renaisse et bientôt un grand récit.

Raison de plus pour interroger l'expression même de grand récit. Aujourd'hui plus que jamais, l'expression prend une couleur péjorative. Pourquoi? Que veut-on dire en renfermant dans ces deux mots grand et récit une polémique?

À l'évidence, le vocable grand est tout spécialement visé. On divisera donc la question : en quoi le grand récit est-il grand ? en quoi a-t-il tort de l'être ? Tout d'abord, on laisse entendre qu'il prétend embrasser la totalité d'un objet; il est grand ensuite parce que son étreinte est censée parvenir, sans perdre la diversité des détails, à ramener l'ensemble sous un point de vue unique, celui de la coupure quasi-topologique que constituerait l'événement ou l'ensemble d'événements résumé par un nom - révolution, par exemple ; il est grand enfin parce que ce point de vue unique prend la forme d'un télos, le plus souvent positif, mais rien n'empêche qu'il soit négatif. Ainsi le grand récit de Rome est, selon Pétrarque, un perpétuel éloge de Rome, parce qu'il choisit comme télos la grandeur ${ }^{22}$; cela étant admis, il pourrait choisir la décadence, sans que cela affecte la structure : Montesquieu en a donné la démonstration dans ses Considérations sur les causes de la grandeur des Romains et de leur décadence. Grâce à ce qui peut passer pour un des premiers exercices de la dialectique historique, le grand récit de la grandeur de Rome et le grand récit de sa décadence ne font qu'un.

Positif ou négatif, le jugement de valeur est essentiel : grandeur de Rome, gravité de sa décadence, importance décisive de la Révolution française - glorieuse selon les uns, calamiteuse selon les autres. L'instant axiologique ne manque donc jamais ; pourtant, il est présenté sous les espèces de la neutralité, grâce à un consensus, supposé donné d'avance. Nul n'est censé douter de la grandeur de Rome, nul n'est censé douter de l'importance de la Révolution française et surtout pas ses adversaires, nul n'est censé douter de la profondeur du retournement galiléen, par quoi les mathématiques quittent l'éternel pour le contingent. Grâce à cela, ceux qui consentent à l'axiologie ne croient pas émettre un jugement de valeur ; ils pensent prendre en compte un donné, comme il est requis d'un historien honnête. À cette lumière, on comprend mieux le geste de Thucydide quand il s'emploie à démontrer l'ampleur de la Guerre du Péloponnèse en la mesurant à l'étalon de la Guerre de Troie. Il fonde un grand récit, qui va d'ailleurs servir de modèle à bien d'autres depuis : celui de la guerre entre deux blocs, qui est aussi une lutte entre démocrates et aristocrates. Or, dans ce cas précis, le jugement de valeur ne lui était pas donné ; il avait à le constituer. De là sa référence à Troie, qui est, elle, un donné admis par tous les Grecs de son temps; de là, la fonction d'initiateur qu'on lui reconnaît: "père de l'histoire scientifique", "père de la géopolitique » etc. De là enfin sa réputation de neutralité; si en effet il accorde une importance majeure à la compétition entre Athènes et Sparte, il ne choisit aucun des deux camps et, quoiqu'Athénien, les malheurs de sa cité ne lui tirent pas une larme. Son 
jugement de valeur ne dépend ni du Bien ni du Mal : il choisit son objet en fonction de son poids quasi-quantitatif dans l'espace de l'oikouménè.

Le plus souvent, cependant, l'évaluation a été menée par une doxa anonyme. Le consensus s'est cristallisé d'avance, au sein d'une communauté sociale, du fait de son imaginaire spontané. En tournant en dérision la grandeur du grand récit, on moque donc la figure du consensus préalable, la forme de la spontanéité doxique et l'absence de pensée. À l'ombre de René Char, Foucault avait érigé en commandement une telle dérision. Il faut pourtant prendre garde : quand le consensus porte sur une rupture, la mise en cause de cette rupture, peut, de manière insidieuse, rétablir le règne de la synonymie. Michel Foucault se réclame de René Char et s'impose le devoir de contredire aux synonymies; soit, mais en niant la différence qui sépare Marx de Ricardo, il rétablit la synonymie au sein de l'économie politique. Il y a plus grave; au cœur des Mots et les choses, la notion d'épistémè repose sur la rupture entre épistémès différentes; par symétrie, elle repose tout autant sur l'absence de rupture interne au sein d'une épistémè donnée. En sorte que Les Mots et les choses établissent des lieux de synonymies, dans la mesure exacte où ils contestent la synonymie comme loi d'ensemble de «toute l'histoire des hommes ». Rien de contradictoire en cela, sauf que le dédoublement du temps épistémique en ruptures et en stases ne suffit pas à disqualifier la forme récit en elle-même. On pourrait avancer au contraire qu'elle la légitime.

\section{5 - La logique de la rupture}

33 Le point de départ d'un récit réside en effet dans la reconnaissance d'une rupture. Il n'est que d'étudier, en linguiste, les incipit des romans, sans omettre de prendre au pied de la lettre le défi lancé par Aragon, Je n'ai jamais appris à écrire ou les Incipit. Le grand récit narre une grande rupture ; le petit récit narre une petite rupture. Sans cela, le récit n'a pas de raison d'être. Quand d'aventure, l'historien s'affranchit de l'exigence, on le range du côté de l'érudition, qui est tenue pour stérile, aussi longtemps qu'elle rencontre des continuités sans fin. Il est d'ailleurs intéressant qu'à l'ombre du grand récit de la Révolution française, soit née et ait prospéré ce qu'on appelle justement la petite histoire; celle-ci privilégie les vies minuscules et montre comment elles persistaient au sein de la discontinuité incessante. La petite histoire marque la revanche des petites continuités sur la grande rupture et celle des petites ruptures sur les grandes continuités.

34 Charles Péguy avait forgé de belles expressions autour du continu et du discontinu. Ainsi présentait-il, en 1911, un recueil de ses propres proses : «Je puis me vanter qu'il n'y a pas dans tout le volume un seul plan incliné; ni une dégradation. Je puis me vanter qu'il n'y a pas dans tout le volume un morceau qui ne commence et qui ne finisse en falaise ${ }^{23}$. On retrouve cette image à plusieurs reprises et toujours laudative ; dans « Un Nouveau théologien M. Fernand Laudet », il écrit à propos d'un communiqué qu'il vient de publier lui-même dans Le Bulletin des professeurs catholiques de l'Université: "Quoi de plus imposant que cette absence de signature au bas du communiqué [...] $\mathrm{Ni}$ titre ni signature. Nulle montée, nulle descente. Nul accès. Nulle porte de sortie. Nul vestibule. Un beau plateau coupé en falaise ${ }^{24}$. » Sous-jacente, on discerne une doctrine : d'un événement qui fait rupture, on ne peut parler qu'en mode de falaise. Or là où il $\mathrm{y} a$ falaise, le récit se brise. 

suivrai Foucault sur un point au moins : tout grand récit doit être soumis à un examen contradictoire. Je suivrai Furet sur un point au moins : il peut arriver qu'en examinant le récit de trop près, on fasse s'évanouir l'événement, mais je lui opposerai aussi une mise en garde : la maxime "rien n'a eu lieu » n'est parfois qu'une variante détournée de la crédulité. Dès qu'on renonce au grand récit hérité, trois voies se proposent :

a. remplacer l'ancien grand récit par un autre, que l'on présentera comme entièrement distinct de tout autre. Il arrive que le nouveau grand récit déplace effectivement l'ancien; ainsi, les grands récits de Michel Foucault, si l'on admet qu'il en a commis. Il arrive aussi que, sous les apparences de l'innovation, on assiste à l'installation d'un grand récit tout aussi ancien, mais appartenant à un autre héritage ${ }^{25}$.

b. abolir la forme du grand récit et lui substituer des séries de micro-récits dont chacun obéit en fait aux mêmes règles de construction que le grand récit, sinon qu'il sera strictement délimité : les plans inclinés et les dégradations s'étendront sur des espaces étroits et clos. Le Dictionnaire critique de François Furet et Mona Ozouf illustre lumineusement cette méthode.

c. renoncer à la forme-récit et s'en tenir à l'effet de falaise : on se propose alors de décrire telle ou telle falaise. Avec mes faibles moyens, j'ai tenté cela pour la Révolution française, en me concentrant sur la Déclaration des droits de 1789 et sur le dernier discours de Robespierre, prononcé le 8 Thermidor. À cette fin, j'ai dû critiquer à la fois la matrice narrative qu'avait élaborée la tradition marxiste-léniniste et certaines tentatives manquées de s'en affranchir ${ }^{26}$.

Je conclurai sur un principe de tolérance : en choisissant la voie (c), je n'ai récusé ni la voie affirmative (a), ni la voie hypercritique (b). Reste que je les juge porteuses d'aveuglement. S'il existe des ruptures dans l'histoire des hommes et si l'on suppose qu'elles sont dicibles, alors elles doivent être dites. Toutefois leur structure fait qu'elles ne peuvent pas être dites sans que le récit rencontre son propre paradoxe : en tant que 
récit, il désire narrer la rupture ; en tant que récit, il demande la continuité qui dénie la rupture. La voie (c) permet de contourner la contradiction, sans renoncer au dire.

\section{NOTES}

1. Alphonse AULARD, Taine historien de la Révolution française, Paris, Armand Colin, 1907. Cet ouvrage rassemble une série d'articles publiés à partir de 1906 dans La Révolution française, revue de la Société de l'Histoire de la Révolution. Aulard y reprend la matière du cours public qu'il venait de donner à la Sorbonne en 1905-1906 et 1906-1907. Le site Gallica donne accès à la revue ; le livre lui-même est consultable sur Internet Archive.

2. Voir, sur ce point, Mona ozouf, Les aveux du roman, Paris, Fayard, 2001. Voir aussi : Aude DÉRUELlE et Jean-Marie ROUlin (dir.), Les Romans de la Révolution, Armand Colin, coll. « Recherches », 2014.

3. Brice PARAIN, Entretiens avec Bernard Pingaud, Paris, Gallimard, 1966, p. 45. Brice Parain attribue le propos à Charles Seignobos, mais sans l'avoir entendu directement.

4. Claude LÉVI-STRAUSS, La Pensée sauvage, Paris, Plon, 1962, p. 336 et suivantes.

5. Christian Jouhaud, Mazarinades : la Fronde des mots, Aubier, collection « Historique ", 1985. Dans sa préface à l'étude sur les 5000 pamphlets politiques, les «mazarinades », Denis Richet insiste sur «cet ensemble disparate d'imprimés allant des vers burlesques aux discours parsemés de citations biblique et de références historiques ».

6. Jean-Marie GOULEMOT, Le règne de l'Histoire. Discours historiques et révolutions. $\mathrm{XVII}^{\mathrm{e}}-\mathrm{XVIII}^{\mathrm{e}}$ siècles, Paris, Albin Michel, 1996.

7. Selon Georges FORESTIER, Molière, Gallimard, 2018, le dernier acte de Tartuffe a été écrit en 1665. On admet que la Fronde se termine en 1653, mais les effets s'en feront sentir longtemps. Ainsi, le Grand Condé n'est définitivement gracié qu'en 1660.

8. La lettre est intitulée «Sur le Parlement »; selon l'édition de Kehl, elle remonte à 1731, mais l'ensemble des Lettres philosophiques a bien été publié en 1734. Ce texte capital a installé dans l'opinion française l'admiration pour le régime parlementaire anglais et la conviction que les guerres civiles anglaises ont réussi, alors que les guerres civiles françaises ont échoué. Voir plus bas ma note 20 pour une version du même récit, modernisée de façon à s'appliquer à la Révolution française et à la naissance des États-Unis.

9. François FURET, Penser la Révolution française, Paris, Gallimard, 1978.

10. François FURET, Le Passé d'une illusion, Calmann Lévy et Robert Laffont, 1995.

11. François FURET, Dictionnaire critique de la Révolution française (dir. avec Mona Ozouf), Paris, Flammarion, 1988.

12. Il est instructif de relire d'une seule traite l'ensemble des notices que Mona Ozouf a rédigées pour le Dictionnaire critique. On y trouve la plupart des éléments constitutifs de la «légende révolutionnaire »: Liberté, Égalité, Fraternité ou le nom Révolution lui-même ; Robespierre mis à part, on y trouve également les figures majeures de cette légende : Danton, Marat et Saint-Just. Ce dernier avait été omis de l'édition initiale de 1988 ; il réapparaît dans l'édition de poche (Paris, Flammarion/Champs, 1992) et cela, est-il écrit dans l'Avertissement, pour répondre aux objections de plusieurs lecteurs. L'article Saint-Just illustre bien l'abord caractéristique de Mona Ozouf : ne pas partir de l'hypothèse que les individus sont médiocres ou les notions, illusoires. L'historienne 
traite la légende révolutionnaire comme une donnée à analyser et non comme une idole à détruire.

13. Le livre de James C. ScotT, Against the Grain (Yale University Press, 2017) a été publié en français sous le titre latin Homo domesticus (La Découverte, 2019). Dans sa préface, Jean-François Demoule mentionne «le grand récit de la naissance de l'État antique ». Dans le cours du texte, excellemment traduit par Marc Saint-Exupéry, l'expression grand récit apparaît plusieurs fois. Or, le plus souvent, l'original anglais parle seulement de narrative. Sauf erreur, l'expression grand narrative y est utilisée une seule fois. La modification introduite par le traducteur rend hommage à l'idiome professionnel des historiens de langue française.

14. Jean-François LYOTARD, La Condition postmoderne, Minuit, 1979.

15. Michel Foucault travaille la Révolution française dans deux cours du Collège de France des années 70 : Michel FoucAult, La Société punitive (1972-1973) et Il faut défendre la société (1976). Il s'exprime brièvement, mais de manière percutante, dans La Volonté de savoir (Gallimard, 1976, p. 117-118). Voir Jacques Guilhaumou, «Michel Foucault et le moment dynastique : de l'Ancien Régime à la Révolution française », dans Écrire l'histoire, n 18, 2018, CNRS Éditions, 2018, p. 89-95. Voir aussi : Sophie WAHNICH, «Michel Foucault et la Révolution française : un malentendu?», dans Id., La Révolution française n'est pas un mythe, Klincksieck, 2017, p. 143-157. Voir enfin la synthèse d'Éric MARTY, Le Sexe des Modernes (Le Seuil, 2021, p. 426).

16. Michel foucAult, Les Mots et les choses, Gallimard, 1966, pp. 86-91. Cette sous-section, intitulée " 'Mathesis' et 'taxinomia' », s'oppose diamétralement à l'approche de Koyré, en établissant une discontinuité radicale entre la science « classique » et la science « moderne ».

17. Ibid., p. 273-274. On est en droit de restituer dans cette sous-section une polémique à peine voilée contre le grand récit althussérien, qui était alors en voie de consolidation.

18. Le qualificatif grand apparaît souvent sous la plume de Foucault. Il recèle généralement une polémique, oscillant entre condamnation et ironie. Dans l'Histoire de la folie, le nom de grand renfermement annonce d'emblée que le lecteur rencontrera de l'intolérable.

19. Alexandre KoYRÉ, Études Newtoniennes, Gallimard, 1968, p. 84.

20. Dans ce qui est présenté comme tome IV de l'Histoire de la sexualité, sous le titre Les Aveux de la chair, Gallimard, 2018, on pourrait interpréter en ce sens la sous-section III du troisième et dernier chapitre. Elle a été intitulée par les éditeurs «La libidinisation du sexe ». Voir en particulier la synthèse finale, p. 372-373. Le christianisme augustinien forme la matrice dont sont issus les discours médiévaux, mais aussi les représentations modernes.

21. Ce point de vue est défendu par Jean-Pierre Dozon, « La fin des grands récits : un diagnostic occidentalo-centré », dans M. Wieworka, L. Lévi-Strauss, G. Lieppe, Penser global, Éditions de la MSH, 2015, p. 259-269.

22. Apologia contra cujusdam Galli calumnias, texte édité et traduit par Rebecca Lenoir sous le titre Invectiva contra eum qui maledixit Italie dans Pétrarque, Invectives, Jérôme Millon, 2003, p. 308.

23. Charles PÉGUY, Cahiers de la Quinzaine, 8e cahier de la douzième série, p. 151. Péguy rend compte du volume Les CEuvres choisies de Charles Péguy 1900-1910, qui vient d'être publié par Grasset. Le bon à tirer du numéro date d'avril 1911. Les Cahiers sont consultables sur Internet Archive, à partir du site http://www.charlespeguy.fr/

24. Cahiers de la Quinzaine, $2^{\mathrm{e}}$ cahier de la treizième série, p. $227, \S 288$. Le bon à tirer date de septembre 1911.

25. Ainsi le grand récit des libertés anglaises et de leur extension aux États-Unis a longtemps été opposé au grand récit de la Révolution française. Guizot a défendu cette cause ; Taine y a adhéré ; alors même qu'aux États-Unis, ce récit, directement issu de Burke, avait été battu en brèche, Hannah ARENDT l'a repris sans réserves dans son grand ouvrage de 1963, On Revolution (Essai sur la Révolution, trad.fr. par Marie Berrane, Gallimard/Folio, 2013). L'intelligence scintille à toutes les pages, mais le renouvellement proposé consiste dans un simple changement d'héritage. 
26. Jean-Claude MILNER, Relire la Révolution, Verdier, 2016. Selon moi, François Furet et Hannah Arendt illustrent, quoique de manière très différente, deux échecs parallèles, face à la « tradition révolutionnaire » qu'ils prétendaient abolir.

\section{RÉSUMÉS}

La Révolution française a suscité très tôt une multiplicité de récits qui ne se bornaient pas à rapporter la totalité des événements, mais tentaient également à en exposer les causes « réelles». Ces récits différaient profondément les uns des autres, mais leur ambition était analogue. Le présent article se propose de montrer que la notion de "grand récit ", introduite par Jean-François Lyotard, permet de saisir les traits communs de ces divers travaux.

On connaît les critiques que François Furet a adressées aux historiens français de la Révolution; elles se laissent résumer comme suit: au lieu de substituer un nouveau grand récit aux récits antérieurs, Furet rejette le grand récit comme tel. De ce point de vue, il s'inscrit dans un mouvement de grande ampleur. Avant lui, Claude Lévi-Strauss avait dressé un parallèle entre les grands récits des sociétés occidentales et les mythes qu'il avait étudiés dans les sociétés amazoniennes. Indépendamment de Furet, Michel Foucault exprime sa défiance à l'encontre des " grands récits » hérités.

Dans ces conditions, une série de questions se pose : les historiens peuvent-ils éliminer toute possibilité de grand récit? Un tel rejet implique-t-il que les micro-récits sont seuls capables de rendre compte des événements passés ? Implique-t-il l'échec inévitable de toute tentative visant à aller au-delà de la pure et simple juxtaposition des descriptions ? Le présent article tente, dans sa conclusion, de résoudre ces difficultés.

The French Revolution gave birth very early to various narratives that intended not only to present the total sequence of events, but tried also to expound on their "real" causes. These narratives differed deeply, but their ambitions were analogous. The present paper intends to show that the common features of such approaches are adequately captured by a notion introduced by Jean-Francois Lyotard, namely the notion of "grand narrative".

François Furet's criticisms of the French historians of the Revolution are well known; they may be summarized as follows: instead of trying to substitute a new grand narrative to the former, he rejected the grand narrative itself. From that point of view, he participated to a wider movement. Before Furet, Claude Lévi-Strauss had drawn an analogy between the grand narratives of Western societies and the myths he studied in the Amazonian societies. Independently of Furet, Michel Foucault expressed his distrust with respect to all inherited "grand narratives".

A series of questions has now to be raised: is it possible for historians to reject completely the form of the grand narrative? Does such a rejection imply that micronarratives are the only possible way of treating past events? Does it imply that all attempts to go further than the mere juxtaposition of descriptions are doomed to fail? In its conclusion, the present paper tries to propound a solution to these difficulties. 
INDEX

Mots-clés : Révolution française, Fronde, grands récits, mythes

Keywords : French Revolution, Fronde, grand narrative, myths 